\title{
mécanisme de déplacement et déformation des nappes exemple de la nappe de Gavarnie (Pyrénées centrales)
}

\author{
par \\ J. Déramond \\ et \\ P. Sirieys \\ Laboratoire de Tectonophysique \\ Université Paul Sabatier, Toulouse
}

\section{Structure géologique}

\section{Les unités}

La nappe de Gavarnie constitue, sur la bordure sud de la zone axiale de la chaîne des Pyrénées, une structure bien définie qui, depuis A. Bresson (1903), a fait l'objet de nombreuses études [1] [2]. Son déplacement du Nord vers le Sud n'est plus à démontrer. On y distingue les unités suivantes (fig. 1 et 2 )

1) La zone de racine, au nord, constituée de terrains sédimentaires uniquement paléozoïques, recoupés de massifs granodioritiques hercyniens affectés par la tectonique alpine [3] [4]. Les structures majeures (stratification, certaines surfaces de schistosité) d'orientation générale $E$. W. sont verticales ou à fort plongement nord [5].

2) La zone centrale, représentée par : i) le substratum métamorphique qui apparaît en fenêtres dans les vallées de Gavarnie, Héas, Barroude; ii) des formations lenticulaires discordantes triasiques et crétacées en couverture normale du substratum; iii) des terrains paléozoïques constituant la partie charriée proprement dite (notée N.P.) recouvrant l'ensemble. Les structures dans la partie charriée sont généralement horizontales ou plongent légèrement vers le Sud. Le passage de la zone de racine à la N.P. se traduit par une courbure des structures (So, schistosités) qui évoluent progressivement de verticales à horizontales.

3) La zone méridionale, caractérisée par la présence, sur le substratum métamorphique et sa couverture discordante, d'une importante série allochtone essentiellement crétacée-éocène reposant sur des formations triasiques d'épaisseur variable (quelques mètres généralement) présentant des décollements importants. Dans cette partie crétacéeéocène de la nappe (notée N.C. E.) la stratification plonge faiblement vers le $S$; au $S$ du Cirque de Gavarnie elle devient horizontale. Les limites de la
N.C.E. sont difficiles à préciser : au sud, le contact de base n'est plus observable à partir du Cirque de Gavarnie, au nord, la partie manquante a été enlevée par l'érosion.

La limite $S$ de la zone de racine est, par contre, définie de façon précise par la courbure de la stratification qui devient horizontale dans la N.P. La limite $N$ de cette zone est beaucoup plus difficile à préciser.

\section{La lithologie}

a) Age des terrains

A l'échelle régionale, la disposition des différents terrains est relativement simple. Dans le Paléozoïque comme dans le Crétacé allochtone, la série stratigraphique ne présente ni redoublement de terrain, ni dispositif inverse; à la base se situent les terrains les plus anciens, au sommet les plus récents. Ainsi, la nappe paléozoïque débute par des formations d'âge silurien et se termine par des terrains carbonifères. De même, dans la partie crétacée-éocène de la nappe, les terrains les plus anciens (albiens, cénomaniens) affleurent à la base, les plus récents (flysch éocène) au sommet. Dans la racine, les terrains les plus anciens affleurent largement dans la partie sud (zone de transition racine-nappe paléozoïque), les termes les plus récents (carbonifères) étant situés plus au nord.

La présence à l'extrémité $S$ de la nappe paléozoïque de terrains carbonifères (lambeaux du Chinipro et de la Estiva) permet d'estimer un déplacement de l'ordre de $15 \mathrm{~km}$ à la partie supérieure de la nappe et de $8 \mathrm{~km}$ à la base (fig. 3).

b) Le facteur de discontinuité stratigraphique

Dans l'ensemble de la série stratigraphique, du fait de l'hétérogénéité lithologique, certains terrains jouent un rôle plus ou moins important dans le déplacement d'ensemble de la structure. Ainsi, dans les formations rythmiques, on observe de nombreuses surfaces de 


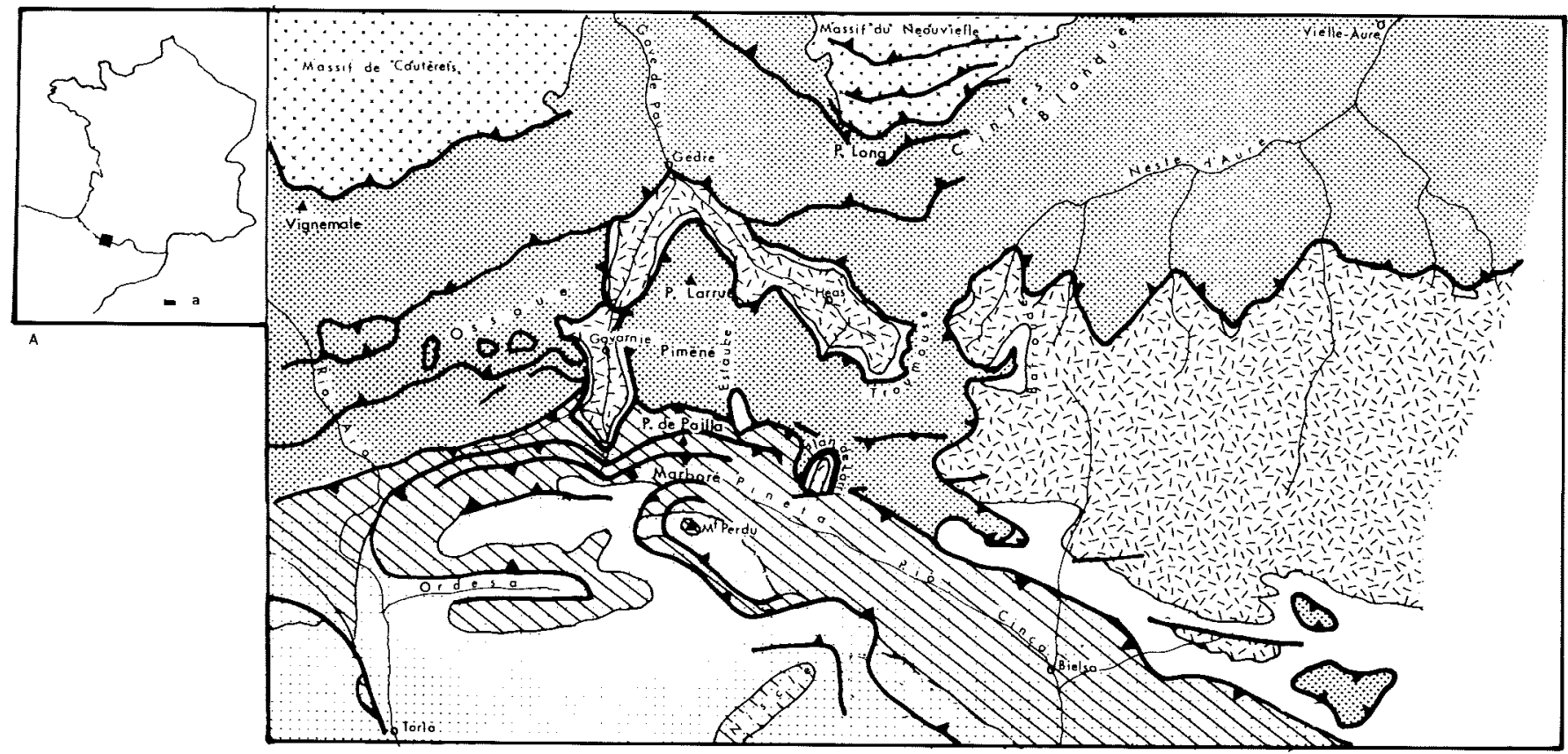

B

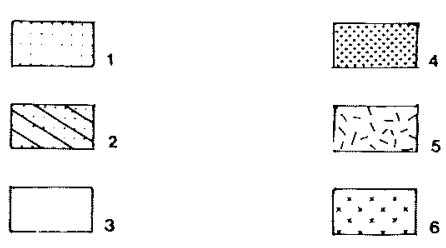

Fig. 1

$A$ Localisation de la zone étudiée

$B$ Carte géologique schématique

1 : Eocène

2 : Crétacé supérieur $\{$ allochtones

3: Trias, crétacé supérieur autochtones

4: Paléozoíque

5 : Socle métamorphique

6 : Massifs "granitiques»

7 : Failles ou chevauchements

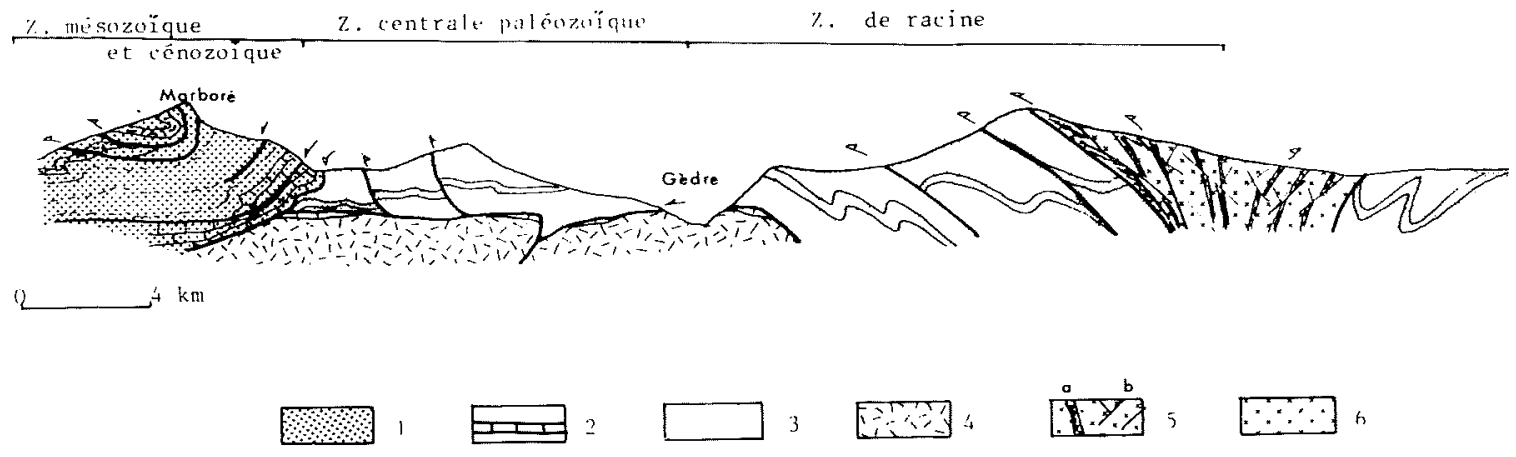

Fig. 2 Coupe NS de la zone étudiée. Définition des divers ensembles géométriques de la nappe de Gavarnie 1 : Crétacé et éocène allochtones (nappe mésozoïque et cénozoïque)

2 : Crétacé autochtone

3 : Paléozoïque

4 : Socle métamorphique

5 : Structures de déformations alpines dans les massifs "granitiques» hercyniens

a) zones de mylonites

b) zones de cisaillement

6 : Roches éruptives (granodiorites) 


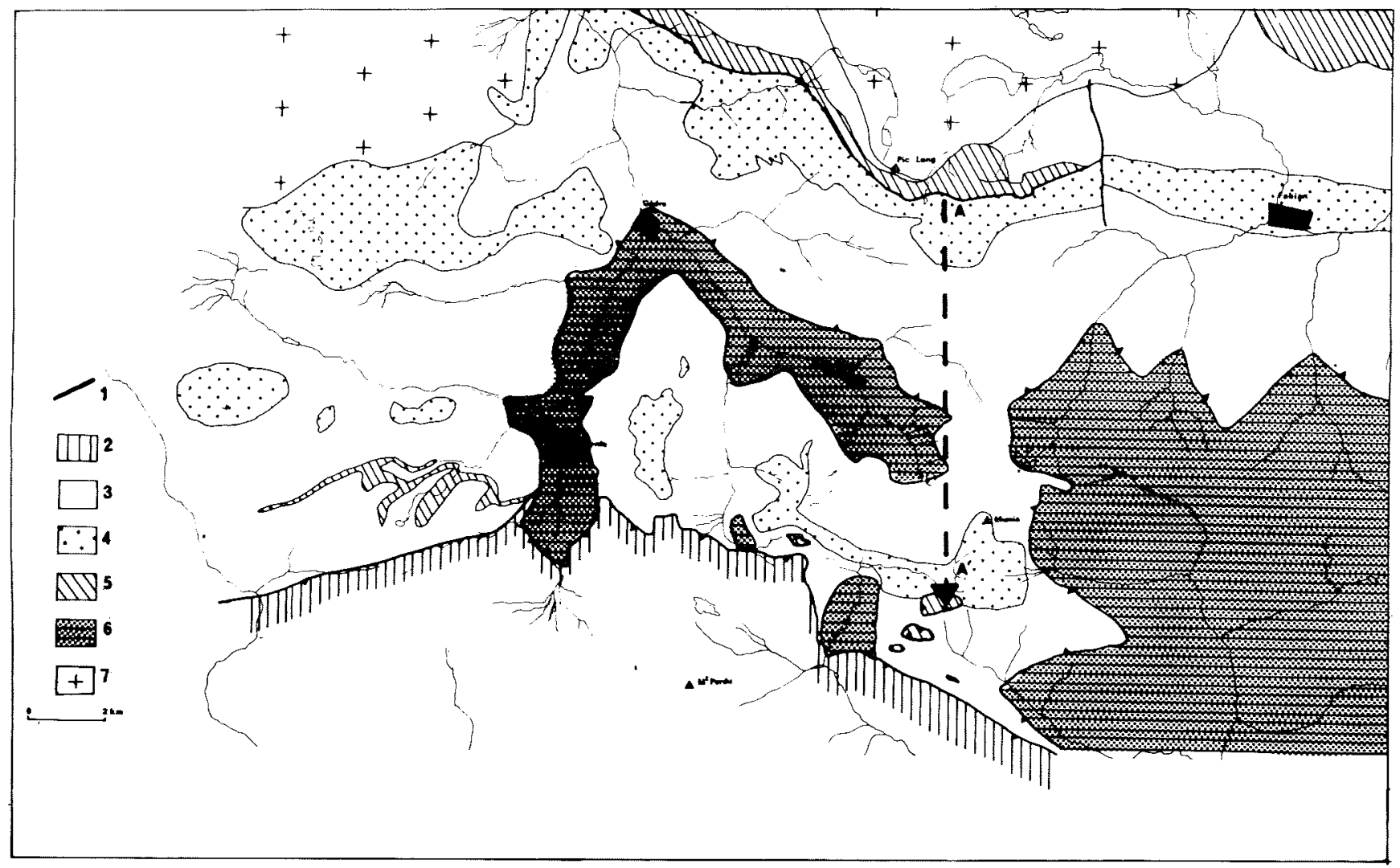

Fig. 3 Estimation du déplacement (AA') dans la nappe paléozoïque

1 : Principales failles

2 : Crétacé-Eocène allochtones

3 : Paléozoïque indifférencié

4 : Dévonien supérieur détritique (série de Sia)

5 : Dévonien supérieur carbonaté

6 : Socle métamorphique

7 : Massifs éruptifs

(le Crétacé autochtone formant la couverture du socle métamorphique n'a pas été figuré ici)

glissement, limitant deux milieux de nature différente. Dans le but d'obtenir un moyen de comparaison entre diverses séries on a représenté la rythmicité, et donc la présence d'éventuelles surfaces de glissement, par le facteur de discontinuité (F.D.) défini comme la fréquence des joints : F. D. $=\frac{n}{E}$ (où $E$ est l'épaisseur totale de terrain considéré et $\mathrm{n}$ le nombre d'intervalles séparant les surfaces de discontinuité éventuelles). Ce facteur permet de comparer, à une échelle donnée, la rythmicité des terrains. Aux fortes valeurs de F.D. correspondent des séries rythmiques avec de nombreuses surfaces de discontinuité; aux faibles valeurs de F.D. correspondent des séries massives où les surfaces de discontinuité sont rares (fig. 4). La détermination systématique du F.D., dans l'ensemble de la nappe, permet de préciser le comportement des différents terrains, lors des déformations alpines.

\section{Les déformations}

Cette nappe est constituée de terrains d'âge varié et de lithologie différente (calcaires massifs, séries rythmiques calcaréo-pélitiques, gréso-pélitiques, calcaréogréseuses, formations pélitiques, ensembles conglomératiques, massifs éruptifs hercyniens intrusifs dans la zone de racine). Sa mise en place est le résultat d'une tectogénèse post-crétacée, comme l'a montré A. Bresson [1] le premier à mettre en évidence le recouvrement anormal du Paléozoïque sur le Crétacé. A la suite des travaux de nombreux auteurs, il apparaît maintenant de façon sûre qu'elle se met en place à l'Éocène, c'est-à-dire au cours de l'orogénèse alpine. La déformation pyrénéenne apparaît donc comme la déformation progressive d'une série stratifiée: le Mésozoïque et le Cénozoïque, et d'un ensemble déjà déformé à l'hercynien : le Paléozoïque.

Structures de déformations superposées. 

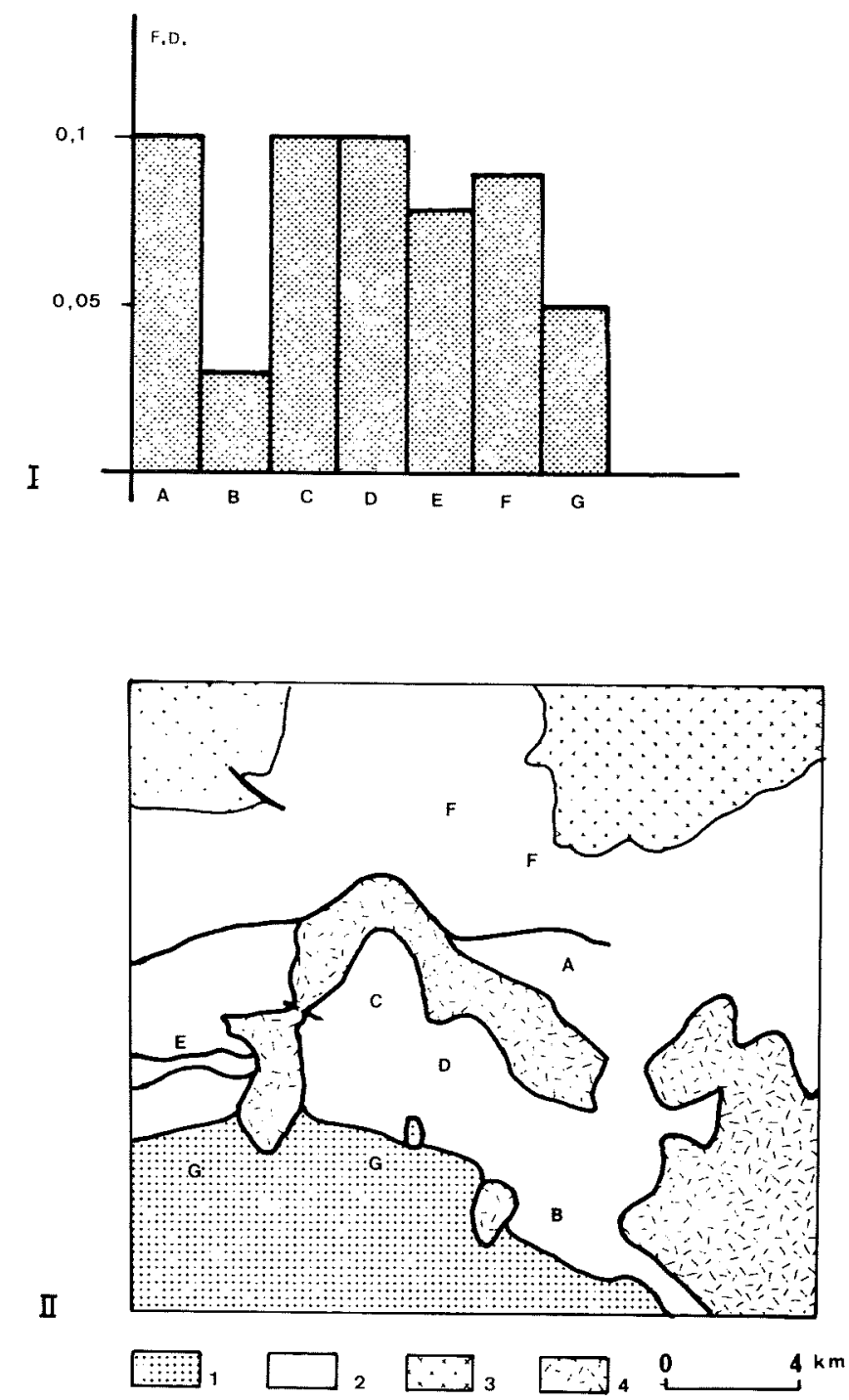

Fig. 4 Valeurs du facteur de discontinuité (FD) mesuré à l'échelle régionale (FD: nombre de discontinuités par mètres).

I Valeurs mesurées

II Localisation des mesures

1 : Crétacé-Eocène allochtone

2 : Paléozoïque

$3:$ :Granites》

4 : Socle métamorphique

On met en évidence les faibles valeurs du FD dans la nappe Crétacé-Eocène $(G)$ et dans la partie $S$ de la nappe paléozoïque $(B)$

D'après les caractères géométriques, on peut distinguer (fig. 5) :

A l'hercynien :

1) Une première déformation hercynienne en plis couchés, visibles exceptionnellement, toujours affectés par les déformations postérieures.

2) Une deuxième déformation hercynienne à schistosité EW, subverticale, plan axial de plis droits au nord dans la zone de racine, horizontale au sud dans la N.P.

3) Des failles hercyniennes, EW elles-aussi, subverticales dans la zone de racine, horizontales dans la zone charriée.
A l'alpin :

4) Des glissements banc sur banc, présentant des stries, dans le Paléozoïque comme dans le Crétacé, qui se produisent dans les zones les plus favorables (surfaces de stratification les mieux individualisées, où le contraste de compétence est important). Ces glissements ont affecté également l'ancienne surface de discordance à la base du Crétacé, ce qui explique les structures particulières (stries et rupture fragile des minéraux) que l'on observe le long de ce contact et surtout sa disposition actuelle par rapport aux surfaces de stratification dans le Paléozoïque. Ces glissements s'accompagnent, dans le C. E. de plis dissymétriques à flanc court tourné vers le $S$ à plan axial orienté $E W$ et à plongement nord, pouvant présenter une charnière cisaillée, leur plan axial étant alors parallèle à So.

5) Des $p / i s$ qui déforment ces structures (plis à plan axial variable, subhorizontal à la base du Crétacé, se redressant ensuite progressivement) dont la schistosité se confond dans le Paléozoïque avec la schistosité hercynienne principale $\left(\mathrm{SH}_{2}\right)$. Dans les lambeaux de Crétacé autochtone, cette schistosité est horizontale et parallèle au contact de base Paléozoïque-Crétacé autochtone de la nappe. Cette schistosité est, en outre, affectée par des failles inverses de direction EW bien développées dans le substratum métamorphique, se poursuivant dans le Crétacé autochtone, mais aussi dans la N.P. ou la N.C.E. Ces failles correspondent au rejeu d'accidents hercyniens qui affectaient les formations migmatitiques et granitiques du socle [6].

6) Des failles EW courbes à concavité tournée vers le $\mathrm{N}$ affectant la N.P.

Finalement, l'étude des structures de déformation dans le Paléozoïque ne révèle pas la présence de grandes structures couchées comme celles que l'on observe dans d'autres nappes (Dent de Morcles, dans les Alpes Valaisannes, par exemple). Les seuls plis couchés visibles sont d'échelle métrique à décamétrique et n'affectent pas la disposition d'ensemble. L'analyse des plis dans le Crétacé montre qu'il n'existe pas non plus de grande structure couchée à l'échelle de la nappe, les grands plis observables sur le terrain se limitent à une ou plusieurs couches. Ces plis, ne déformant que certaines assises, sont appelés plis internes.

Ces caractères géométriques (notamment absence de grands plis couchés à l'échelle de la nappe et présence d'un chevauchement à la base) amènent à considérer chacun des deux ensembles superposés (N.P. et N.C.E.) comme une nappe de chevauchement.

Dans le Mésozoïque et le Cénozoïque, les formations concernées présentent en général, un faible F. D. c'està-dire un petit nombre de surfaces de glissement effectives d'importance régionale. Les structures de déformation entre deux surfaces de glissement sont des $p$ lis dissymétriques à flanc court tourné vers le sud ou des plis à charnières cisaillées; ces structures (surface de glissement, plis dissymétriques, plis à charnières cisaillées) sont déformées postérieurement avec une schistosité courbe, subhorizontale à la base de la série mésozoïque, à plongement nord $\left(20\right.$ à $\left.25^{\circ}\right)$ à la partie supérieure.

Dans le Paléozoïque, le F.D. est en général fort. Accidentellement, le glissement peut se produire suivant les surfaces de schistosité hercyniennes (ce 


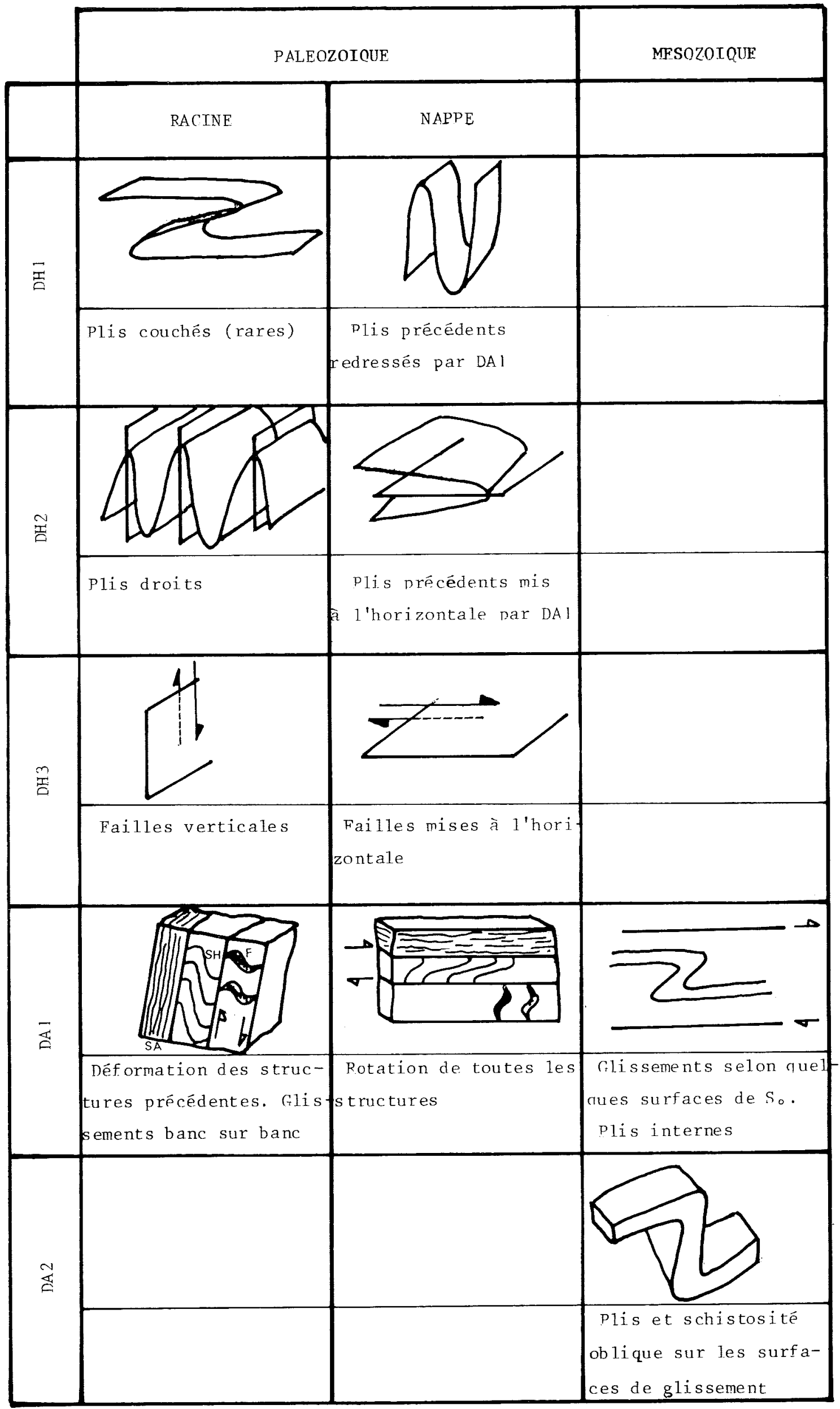

Fig. 5 Superposition des déformations : structures induites 
que l'on observe dans les charnières de plis). De manière générale, le glissement banc sur banc est rendu visible par la déformation de surfaces antérieures (schistosité hercynienne $\mathrm{SH}_{2}$ ) dans les lits incompétents, par la présence de stries de glissement dans les joints de stratification et par la présence de fentes d'extension rectilignes perpendiculaires à So dans les lits compétents; ces fentes peuvent être affectées localement par un cisaillement simple, parallèle à So.

Dans les massifs éruptifs plus rigides [3] [4], existent deux types de structures de déformation, des zones de mylonites, les plus anciennes, et des zones de cisaillement. Dans l'encaissant du massif, les zones de mylonites se poursuivent, à l'est comme à l'ouest, par des failles inverses subverticales confondues avec la stratification. Loin du massif, elles sont remplacées par des glissements NS plus discrets suivant So. Ces observations amènent à supposer que les zones de mylonites existaient déjà lors de la mise en place de la nappe; elles seraient donc probablement hercyniennes, du moins pour une partie d'entre elles. Les zones de cisaillement ne provoquent pas de déformation notable dans l'encaissant du massif plutonique. Des structures équivalentes n'existent ni dans le Paléozoïque, ni dans le Crétacé.

Le comportement au cours de la déformation pyrénéenne des divers ensembles constituant la nappe de Gavarnie s.1. fonction de la rythmicité des séries et donc de la présence ou de l'absence de surfaces nettes séparant des lits de compétence différente.

\section{Détermination de la déformation}

Les résultats des mesures de déformation permettent de mettre en évidence, dans le Paléozoïque (par comparaison avec d'autres zones de la chaîne réputées peu déformées lors de l'orogénèse pyrénéenne) l'importance respective de la déformation alpine et de la déformation hercynienne, et en outre les relations entre déformation continue et déformation discontinue, c'est-à-dire de préciser le modèle de mise en place d'une telle structure.

Les objets ellipsoïdaux tels que fossiles et galets, sont les témoins les plus abondants et les plus largement répartis dans l'ensemble de la structure [7]. Suivant les relations de compétence entre les marqueurs et leur matrice, la détermination du taux de déformation a été conduite à partir de deux analyses : la répartition des populations déformées, lorsque marqueurs et matrice ont même comportement [8] ou le boudinage, lorsque les objets sont compétents par rapport à leur matrice et affectés par des fentes. A partir des mesures d'orientation et de longueur des fibres minérales qui cristallisent dans des fentes d'extension ou dans des zones d'ombres de pressions, il est possible de déterminer la valeur de l'incrément de déformation pour une orientation de fibre donnée [9] [10] c'est-àdire de déterminer la déformation infinitésimale associée à la déformation finie.

Les résultats des mesures de déformation effectuées en 33 points de la nappe, localisés sur la figure 7 , sont résumés dans le tableau 1 , dans lequel les différents points de mesures ont été regroupés selon leur position à l'intérieur de la nappe s.l. (zone de racine, partie centrale autochtone, Mésozoïque allochtone). Pour la plupart des points de mesures les marqueurs utilisés avaient même compétence que la matrice, pour les points $20,22,24$, les objets déformés étant des grains minéraux fragmentés par boudinage, leur état initial a pu être déterminé par reconstruction comme pour un puzzle. Ces mesures nous ont permis, en considérant l'invariance de volume, de déterminer en chaque point les composantes principales de la déformation finie, figurées dans le tableau 1 sous forme de déformation logarithmique $\bar{e}_{1}, \bar{e}_{2}, \bar{e}_{3}$ (on passe de la déformation logarithmique $\overline{\mathrm{e}}$ à la déformation linéaire $\in$ par la relation $\overline{\mathrm{e}}=\log (1+\in)$ ). Le signe de la déformation intermédiaire $\overline{\mathrm{e}}_{2}$ indique la nature de la déformation :

$\overline{\mathrm{e}}_{2}<0$ la déformation est fibreuse (dite aussi par constriction) son ellipsoïde est allongé ou en «cigare».

$\bar{e}_{2}>0$ la déformation est schisteuse (dite aussi par aplatissement) son ellipsoïde est aplati ou en "galette».

L'orientation de la déformation est caractérisée par celle de son trièdre principal $X, Y, Z$ correspondant respectivement à $\overline{\mathrm{e}}_{1}, \overline{\mathrm{e}}_{2}$ et $\overline{\mathrm{e}}_{3}$. L'orientation des plans $X Y(70$ à $130 \mathrm{~N})$ et les taux de déformation mesurés montrent que, dans le Paléozoïque, la déformation résulte d'un aplatissement NS observable en zone de racine (cf. § 3). Dans la N.C.E., l'hétérogénéité de la déformation manifestée notamment par la courbure de la schistosité paraît jouer un rôle important lors de la mise en équilibre de la nappe (schistosité courbe, avec un taux de déformation plus important à la base).

La comparaison des résultats obtenus dans la nappe de Gavarnie avec ceux enregistrés plus au nord, dans les zones où la déformation pyrénéenne est faible, montrent que cette dernière se manifeste par une déformation "interne" (c'est-à-dire entre deux surfaces de discontinuité) peu intense des roches, alors que les déplacements importants du nord vers le sud de la nappe sont dus à la tectonique alpine. Effectivement, [11] la plus grande part des déplacements est de nature discontinue, par glissement discontinu banc sur banc.

Ces résultats permettent de donner le schéma suivant de mise en place de la nappe : raccourcissement NS, responsable de la formation de failles inverses dans le socle et de glissements le long des surfaces de stratification dans les terrains paléozoïques, et déplacement vertical ascendant dans la zone de racine des terrains paléozoïques et horizontal vers le sud où ils recouvrent le socle migmatitique. La montée, plus importante au Nord qu'au Sud, se traduit par un soulèvement moins intense des massifs éruptifs plus compétents que des terrains qui les entourent, il se crée, finalement, une pente suivant laquelle le Crétacé sus-jacent va se déplacer vers le Sud.

\section{Mécanismes de déformations pyrénéennes (Alpines)}

Les résultats de mesures montrent donc qu'il existe dans la nappe deux types de déformation liés à sa mise en place:

- Une déformation continue hétérogène suivant les diverses unités, de type déformation pure par raccourcissement perpendiculaire aux couches dans le Paléozoïque (racine et N.P.) et le Mésozoïque autochtone associée à une déformation rotationnelle de type glissement hétérogène, important dans la N.C.E.

- Une déformation discontinue résultant d'une translation banc sur banc suivant les surfaces de 
Tableau 1

Résultats de mesure

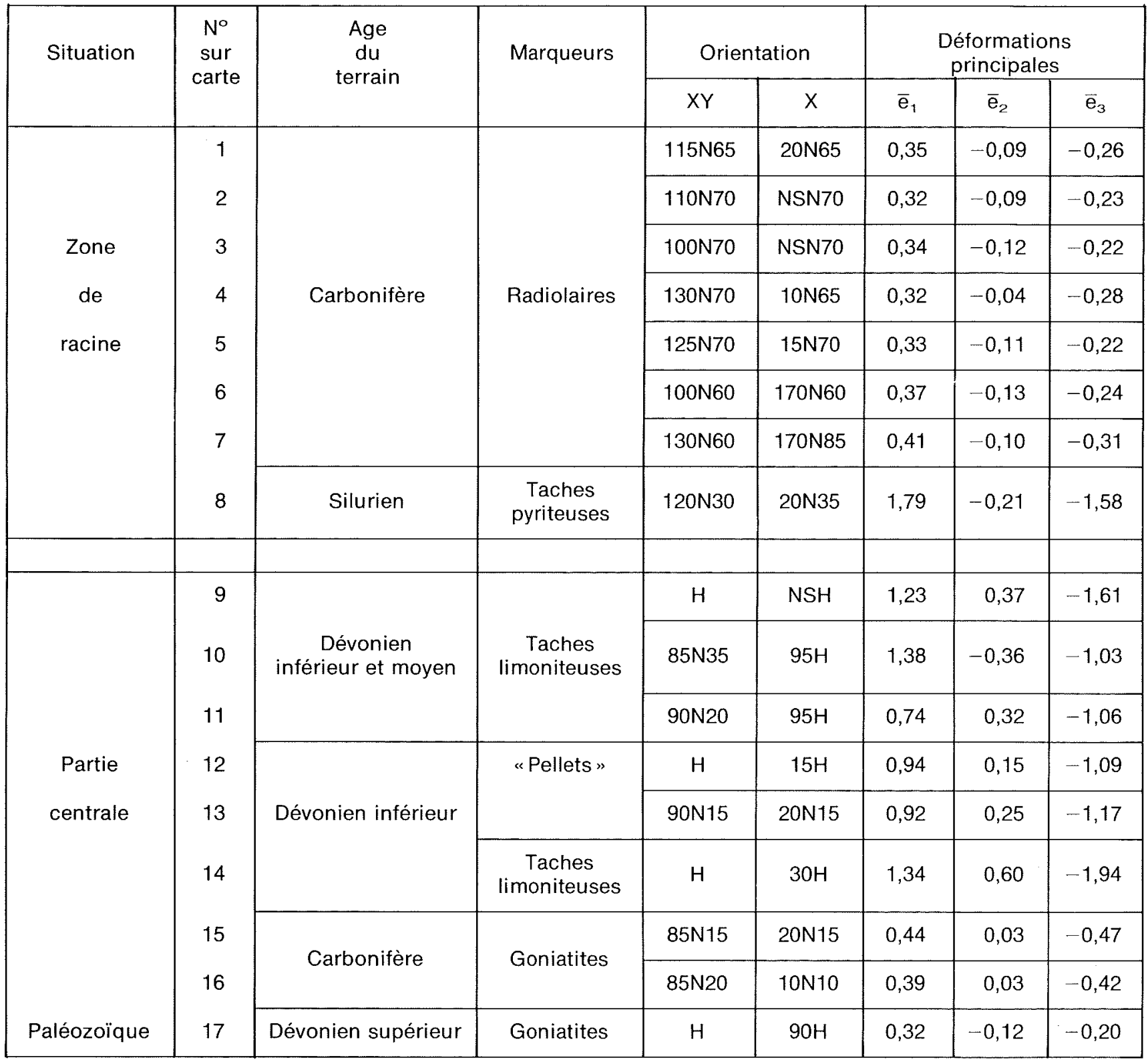

Suite du tableau 1 
Tableau 1

Résultats de mesures (suite)

\begin{tabular}{|c|c|c|c|c|c|c|c|c|}
\hline \multirow[t]{2}{*}{ Situation } & \multirow[t]{2}{*}{$\begin{array}{l}\mathrm{N}^{\mathrm{O}} \\
\text { sur } \\
\text { carte }\end{array}$} & \multirow[t]{2}{*}{$\begin{array}{l}\text { Age } \\
\text { du } \\
\text { terrain }\end{array}$} & \multirow[t]{2}{*}{ Marqueurs } & \multicolumn{2}{|c|}{ Orientation } & \multicolumn{3}{|c|}{$\begin{array}{l}\text { Déformations } \\
\text { principales }\end{array}$} \\
\hline & & & & $X Y$ & $x$ & $\bar{e}_{1}$ & $\overline{\mathrm{e}}_{2}$ & $\bar{e}_{3}$ \\
\hline \multirow{7}{*}{ Autochtone } & 18 & Crétacé & Quartz & $70 N 10$ & $170 N 10$ & 0,30 & $-0,09$ & $-0,21$ \\
\hline & 19 & Trias & Galets & $80 N 10$ & 170 N20 & 0,56 & $-0,16$ & $-0,40$ \\
\hline & 20 & \multirow{2}{*}{ Crétacé } & Puzzle & 110 N30 & 20 N35 & 0,53 & 0,39 & $-0,93$ \\
\hline & 21 & & Galets & 90N15 & $175 N S$ & 0,52 & 0,42 & $-0,94$ \\
\hline & 22 & & Puzzle & & & 0,51 & 0,44 & $-0,95$ \\
\hline & 23 & Trias & Galets & $\mathrm{H}$ & $90 \mathrm{H}$ & 0,97 & $-0,20$ & $-0,76$ \\
\hline & 24 & Crétacé & Puzzle & $\mathrm{H}$ & $15 \mathrm{H}$ & 0,55 & 0,13 & $-0,68$ \\
\hline \multirow{6}{*}{ Mésozoıque } & & \multirow{3}{*}{ Trias } & \multirow{3}{*}{ Galets } & & & & & \\
\hline & 25 & & & $75 \mathrm{~N} 10$ & $25 \mathrm{~N} 20$ & 0,45 & 0,34 & $-0,79$ \\
\hline & 26 & & & $70 \mathrm{~N} 15$ & 15N15 & 0,44 & 0,25 & $-0,68$ \\
\hline & 27 & $\begin{array}{c}\text { Crétacé } \\
\text { Cénomanien }\end{array}$ & $\begin{array}{l}\text { Dragées } \\
\text { Quartz }\end{array}$ & $85 \mathrm{~N} 15$ & NSN10 & 0,60 & 0,21 & $-0,81$ \\
\hline & 28 & \multirow{2}{*}{ Santonien } & Foraminifères & $\mathrm{H}$ & $10 \mathrm{H}$ & 0,56 & 0,15 & $-0,71$ \\
\hline & 29 & & + «Pellets" & $90 N 10$ & $20 N 10$ & 0,16 & 0,04 & $-0,20$ \\
\hline \multirow{3}{*}{ Allochtone } & 30 & \multirow{3}{*}{$\begin{array}{l}\text { Campano- } \\
\text { Maestrichtien }\end{array}$} & \multirow{3}{*}{ Foraminifères } & 100 N35 & $80 \mathrm{H}$ & 0,42 & 0,06 & $-0,48$ \\
\hline & 31 & & & $110 \mathrm{~N} 40$ & 80E20 & 0,39 & 0,07 & $-0,46$ \\
\hline & 32 & & & $105 \mathrm{~N} 40$ & $90 \mathrm{H}$ & 0,46 & 0,07 & $-0,52$ \\
\hline
\end{tabular}

So. La fréquence des surfaces de discontinuité dépend de la rythmicité, donc de l'hétérogénéité, des séries sédimentaires déformées. Rappelons que dans le Paléozoïque, le domaine d'homogénéité est généralement très restreint, d'où une multitude de surfaces de glissement, en revanche, dans le Mésozoïque et le Cénozoïque, ce domaine peut devenir très grand et se traduire par des surfaces de glissement très éloignées les unes des autres. A cette déformation discontinue sont localement associées des structures de déformation interne différentes suivant la fréquence des plans de glissement (F.D.).

\subsection{Le frettage}

Dans le Paléozoïque, où les plans de glissement sont très rapprochés, on observe, dans les bancs incompétents, au contact des bancs compétents adjacents, des bandes de glissement de faible épaisseur où l'allure des marqueurs (surface de schistosité) traduit une déformation par glissement hétérogène, semblable à celle décrite par Ramsay et Graham [12]. Dans les bancs compétents apparaissent des fentes, initialement rectilignes, distordues, caractéristiques d'un cisaillement hétérogène superposé à un cisaillement simple. Cette dualité de structures dans les bancs adjacents a conduit à envisager un mécanisme (fig. 6) particulier de déformation d'ensemble [13] caractérisé d'une part par une déformation pure de type raccourcissement perpendiculaire aux couches, en dehors des bandes de cisaillement, marqué, dans les lits compétents, par des fentes ou des fractures conjuguées et parfois dans les bancs incompétents, par une schistosité plane parallèle à So, d'autre part, dans des bandes, au contact des lits compétents, par un glissement hétérogène. Ce mécanisme de déformation dans les terrains paléozoïques (déformation pure globale provoquant un cisaillement simple local) caractérise un frettage de tête, bien connu en mécanique expérimentale des roches, les séries rythmiques pouvant être schématisées par un modèle constitué d'un empilement d'un grand nombre de couches planes, d'épaisseur variable, constituées de matériaux rigides pour les lits compétents et plastiques pour les lits incompétents. Dans une couche plastique, un segment de droite caractérisant la trace des surfaces de la schistosité hercynienne principale, (initialement incliné de $\alpha$ sur l'axe d'aplatissement $O x$ ) se transforme en une courbe composée dans la zone d'aplatissement pur, d'un segment de droite (incliné de $\alpha$ ' sur $O x$ ) et, dans la zone frettée, d'une portion de parabole. 

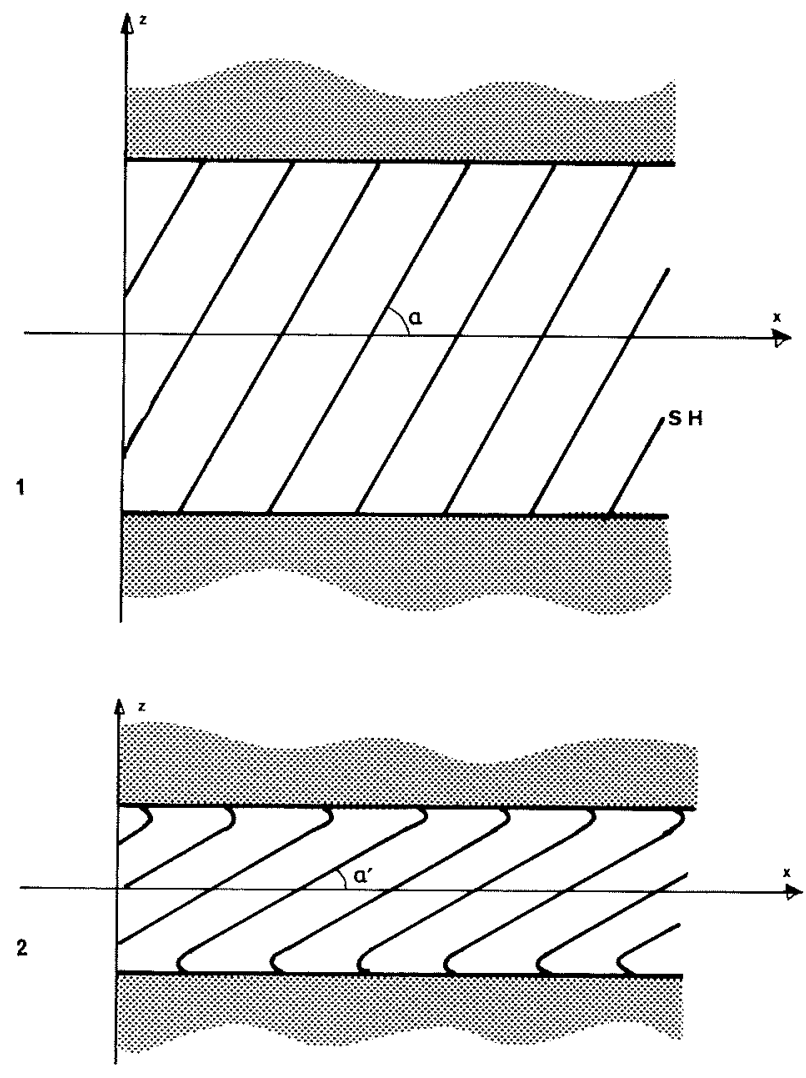

Fig. 6 Frettage

1 : Etat initial (SH : surface hercynienne)

$2:$ Etat déformé

A l'intérieur d'une même couche, on observe, en outre, une évolution des structures indiquant un amortissement latéral progressif, suivi d'une nouvelle zone où la déformation par frettage devient intense et ainsi de suite.

A l'échelle de la nappe, on peut ainsi expliquer par frettage, la déformation locale importante que subissent certaines formations plastiques au contact de formations rigides (pélites siluriennes et substratum métamorphique).

\subsection{Les plis}

Dans le Mésozoïque et le Cénozoïque, entre deux surfaces de glissement, se développent des plis dissymétriques à flanc court tourné vers le sud, l'angle entre leur surface axiale et les surfaces de glissement étant de l'ordre de $35^{\circ}$ à $40^{\circ}$, ainsi que des plis dissymétriques dont le flanc est cisaillé, la surface de cisaillement étant souvent une surface de glissement majeure. Ces deux types de plis procèdent du même mécanisme d'initiation, les plis à flanc cisaillé représentant un stade plus évolué de ces plis dissymétriques.

$\mathrm{Au}$ cours d'une déformation par glissement simple parallèlement aux surfaces de stratification, il peut se produire une hétérogénéité du déplacement qui va se traduire par un flambement des couches. Cette hétérogénéité (en quelque sorte un "blocage" du déplacement) peut être due à la variation du contraste de compétence le long d'un joint de stratification, à la présence d'accidents sédimentaires (variations latérales de faciès) ou à des variations locales de géométrie. Une fois le pli initié par flambement plusieurs mécanismes de déformation peuvent apparaître, suivant la rigidité relative des couches, lui conférant les caractères observés sur le terrain:

1) Faible contraste de compétence : le flanc court du pli passe progressivement du champ de raccourcissement au champ d'extension avec amincissement important (fig. 9).

2) Fort contraste de compétence : il apparaît des glissements inversés d'un flanc à l'autre, beaucoup plus importants dans le flanc court que dans le flanc long (fig. 8) atteignant la rupture fragile; deux surfaces de rupture de type Riedel peuvent se créer mais souvent une seule $F_{2}$ se manifeste (fig. 8). Dans certains cas, cette surface $F_{2}$ peut prendre un développement tel qu'elle atteint la surface de glissement majeur; si la discontinuité le long de cette surface $F_{2}$ est importante, la charnière du pli peut même se déplacer jusqu'à cette surface. Dans d'autres cas, plusieurs fractures parallèles à $F_{2}$ peuvent se développer dans le flanc court du pli et subir une rotation au cours de la déformation progressive. La déformation responsable de la création de ces plis est relativement faible, le taux de glissement (gradient de déplacement dans la direction normale au glissement) est de l'ordre de 0,5 .

Ces plis sont ensuite déformés par une schistosité courbe, horizontale à la base de la N.C.E., se redressant vers le haut, résultant d'une déformation rotationnelle hétérogène.

\subsection{Influence de la structure (anisotropie)}

Le comportement anisotrope des terrains constituant la nappe dépend des surfaces structurales essentiellement par trois facteurs: i) leur fréquence; ii) leur orientation (par rapport à la déformation, donc aux contraintes); iii) les constantes rhéologiques sur ces surfaces, c'est-à-dire l'adhérence. Ces trois facteurs pouvant intervenir seuls ou associés, ont une influence sur la mise en place de la nappe qui s'effectue par glissement le long de telles surfaces (préexistantes).

1) Fréquence des surfaces d'anisotropie (fig. 10)

Dans le Paléozoïque, à plans de glissement nombreux et rapprochés, la déformation à l'échelle de la nappe est assimilable à un flux plastique, l'écoulement correspondant au glissement selon les nombreux plans de stratification, la mise en place de la N.P. se fait par fluage [14] [15] [16].

En revanche, dans la N.C. E. à plans de glissement peu nombreux, la mise en place de la N.C.E. se fait par glissements localisés. [17] [18].

2) Orientation des structures antérieures

Dans les charnières de plis hercyniens, les surfaces de schistosité $\mathrm{SH}_{2}$ plan axial se substituent en tant que surfaces de glissement, aux surfaces de So.

Dans la N.C.E., le glissement gravitaire se produit lorsque les surfaces de stratification sont suffisamment inclinées (du fait de la création d'une pente au toit du Paléozoïque lors de la mise en place de la N.P.). Dans la partie de la N.C.E. reposant directement sur le 


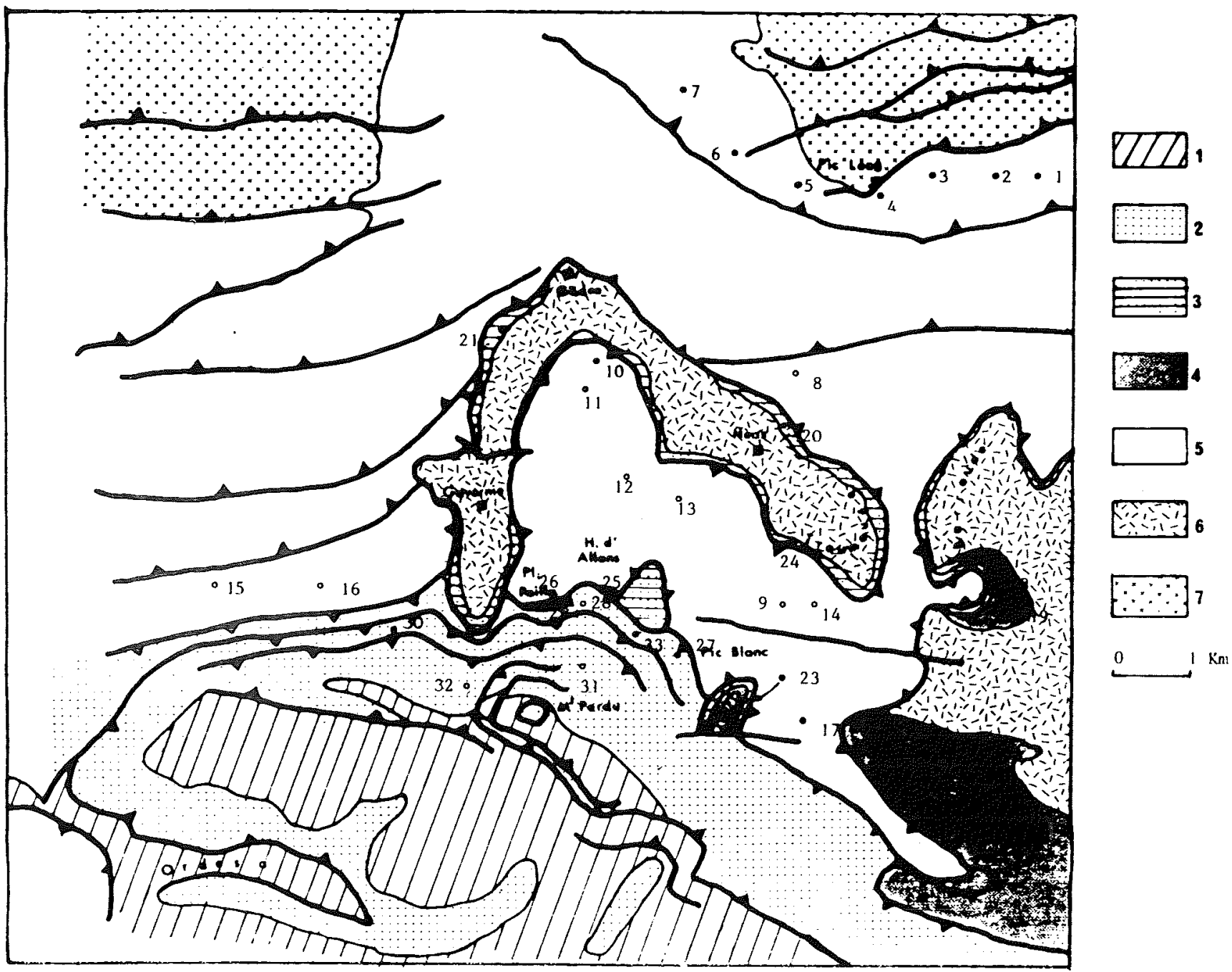

Fig. 7 Localisation des mesures de déformation (chiffres sur la carte) Légende : voir figure 1

Fig. 8 Séquences de la déformation : cisaillement, formation de plis internes, failles liées aux plis par cisaillement

A Déformation infinitésimale

$S$ : schistosité à $45^{\circ}$ de la direction de cisaillement

1 et 2 : surfaces de Riedel

B Formation d'un pli dans un cisaillement général, cisaillement mineur dans les flancs; le cisaillement est plus important dans le flanc le plus court

C Apparition de plusieurs surfaces de Riedel parallèles dans le flanc du pli. Une seule direction de fracture, cor-

rectement orientée par rapport au cisaillement général se développe (direction 2)

$D, E \quad$ Evolution du pli présentant plusieurs surfaces de Riedel dans le flanc court

$C^{\prime}, D^{\prime}, E^{\prime}$ Evolution des plis dans le cas d'une surface de fracture 



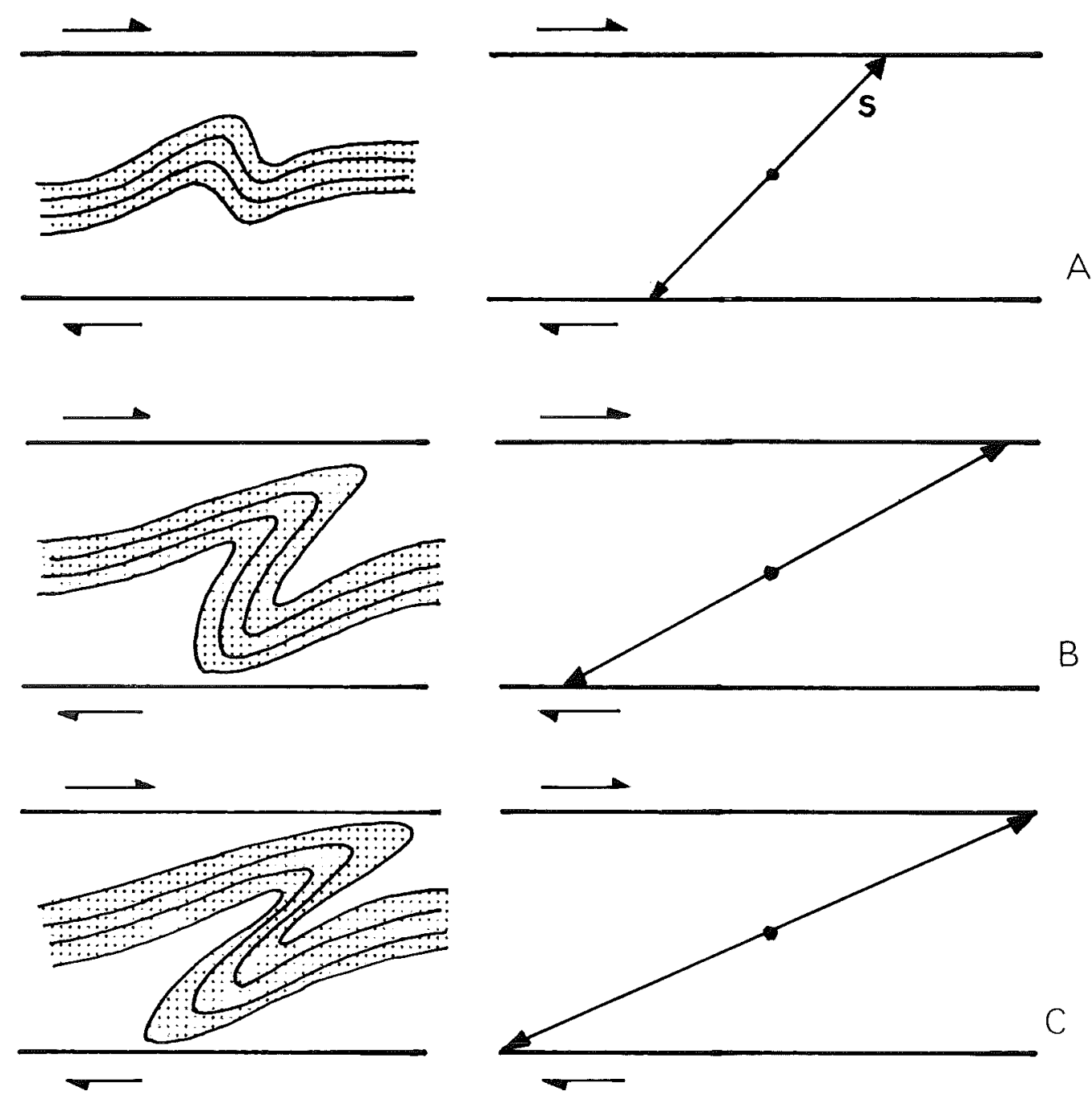

Fig. 9 Evolution d'un pli interne dans un cisaillement. Les couches plissées sont considérées comme incompétentes. A, $B$, $C$, divers stades de l'évolution. S : schistosité
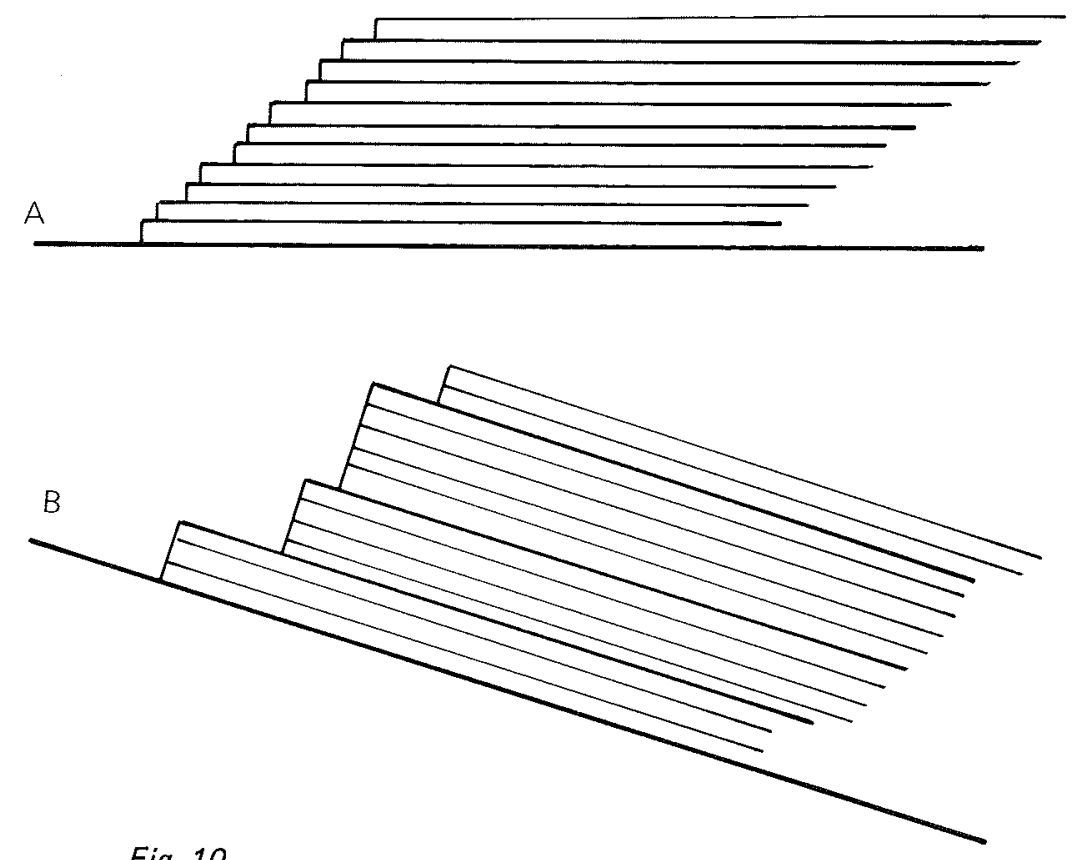

Fig. 10

A Schématisation du Paléozoïque (grand nombre de surfaces de glissement actives)

B Schématisation du Crétacé-Eocène (petit nombre de surfaces de glissement actives) 
substratum métamorphique ou sa couverture, les surfaces de So sont horizontales, un glissement suivant So s'effectue par suite de l'action du bloc sur la pente descendante.

\section{3) Adhérence}

Plusieurs exemples montrent l'influence de l'adhérence suivant les surfaces de stratification dans le Paléozoïque; la surface de schistosité hercynienne $\mathrm{SH}_{2}$ fait un angle faible avec So; parfois cette surface $\mathrm{SH}_{2}$ occupe une position nettement plus favorable au glissement que So; mais ce n'est qu'exceptionnellement qu'il se produit suivant $\mathrm{SH}_{2}$ (charnières de plis hercyniens), car So est une surface de glissement facile par rapport à $\mathrm{SH}_{2}$.

L'adhérence varie le long des surfaces So dans la N.C.E. (comme observé ci-dessus) et peut se traduire par un «blocage» local du glissement responsable de l'initiation de plis (liés au cisaillement).

L'adhérence varie également le long de la schistosité hercynienne principale dans le substratum métamorphique en fonction de la lithologie; le substratum est, en effet, hétérogène (migmatites, granitoïdes, micaschistes, gneiss). La déformation alpine se traduit par la création de failles inverses empruntant la schistosité hercynienne $\mathrm{SH}_{2}$, nombreuses, rapprochées et peu visibles dans les micaschistes, par contre, d'importance régionale dans les migmatites et les granites.

\section{Modèles de mise en place}

Dans le cas de plans de glissement inclinés, faisant un angle $\theta$ avec l'horizontale, le glissement s'effectue lorsque le seuil de frottement le long de la surface de rupture est atteint, c'est-à-dire lorsque la contrainte de cisaillement sur ce plan, égale à : $\tau_{x y}=\gamma h \sin \theta$ $(\gamma$, poids volumique; $h$, épaisseur de roche en mouvement) vérifiera la condition de plasticité. En supposant les plans de glissement très rapprochés et le milieu homogène, on se trouve dans les conditions d'un fluage plan, tel qu'il a été défini par Nye [20] [21] dans les glaciers, assimilable à un demi-modèle de Prandlt. Dans un tel modèle, lorsque la condition de plasticité de type: $\left|\tau_{\max }\right|=\mathrm{K}$ (in Nadaï, [22]) est satisfaite (les valeurs absolues des contraintes cisaillantes atteignent leur maximum $\mathrm{K}$ ), les lignes de cisaillement maximum forment un réseau de cycloïdes orthogonales (fig. 11). II existe [22] deux solutions au problème de la déformation d'un tel modèle dont la différence se caractérise par l'orientation des cycloïdes : Iorsqu'elles tournent leur concavité dans le sens inverse du déplacement, le fluage est dit actif; lorsqu'elles la tournent dans le sens du déplacement, le fluage est passif. Pour le fluage passif, les surfaces limites du modèle ont tendance à se rapprocher; elles s'écartent pour un fluage actif [23] (fig. 11).

\section{Le Paléozoïque}

Dans la N.P. on a observé (cf. § 2), d'une part, un glissement le long des surfaces de stratification parallèles au contact de base de la nappe, d'autre part, des failles inverses d'importance régionale, à concavité tournée vers le nord. Les séries paléozoïques rythmiques à lits de faible épaisseur pouvant être considérées comme des ensembles homogènes se déformant plastiquement, leur mise en place peut être schématisée par un modèle de Prandlt; les deux plateaux rigides du modèle étant représentés, à la base de la N.P. par le
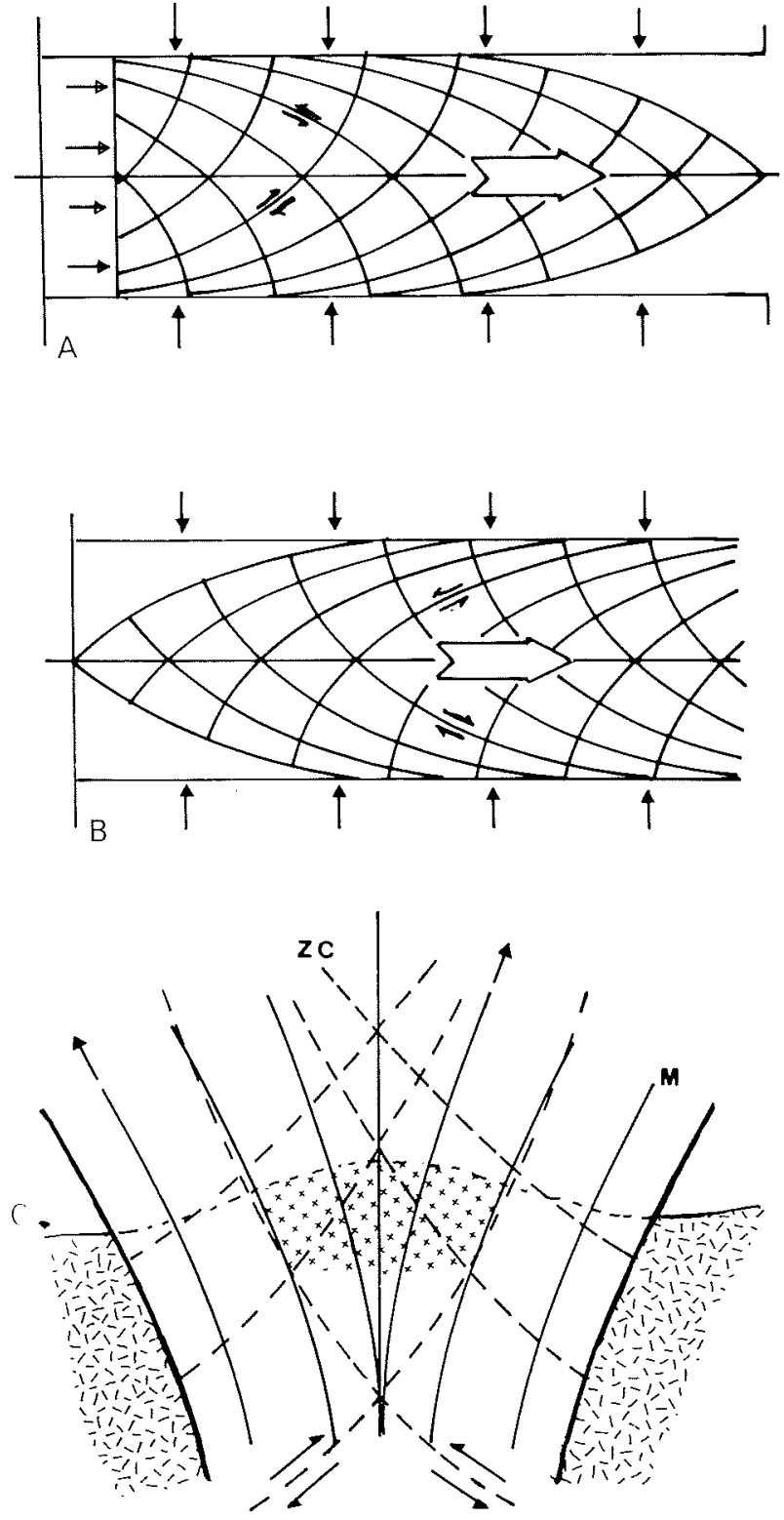

Fig. 11 Modèle de mise en place de la nappe paléozö̈que

A Modèle de Prandtl actif

B Modèle de Prandtl passif

$C$ Zone de racine paléozoïque

$M$ : Mylonites ; $Z C$ : zone de cisaillement; tirets : socle métamorphique ; croix : "granites»

substratum métamorphique, à la partie supérieure par l'ensemble Crétacé-Éocène. Dans le détail, le modèle est plus complexe du fait de la présence de formations calcaires rigides et du déplacement vers le sud du Crétacé-Éocène (c'est-à-dire du glissement du plațeau supérieur).

Dans la zone de racine, on peut envisager un modèle semblable, compliqué par la présence des massifs plutoniques dans lesquels se développent des zones de cisaillement parallèles aux lignes de cisaillement maximum (fig. $11 \mathrm{c}$ ) et qui interviennent comme corps relativement rigides dans la déformation des terrains sédimentaires. 
Le Paléozoïque de la nappe de Gavarnie peut donc être assimilé à deux modèles de Prandlt en relais; le premier, vertical, correspond à la zone de racine, le deuxième, horizontal, correspond à la N.P. Dans le détail, dans chacun de ces deux modèles essentiels, il conviendrait d'envisager des modèles secondaires montrant l'action des formations compétentes existant dans cette série et pour la partie sud le déplacement tangentiel du C.E.

\section{Le Crétacé-Éocène}

L'ensemble Crétacé-Éocène allochtone repose (cf. § 1.3) sur la nappe paléozoïque ou sur le mésozoïque autochtone par l'intermédiaire d'un contact anormal jalonné de lambeaux de formations permo-triasiques (grès, conglomérats, argiles). La disposition en lentilles de ces terrains, le comportement des formations permo-triasiques, l'absence de surfaces de rupture importante dans ces formations (bien qu'existent les preuves manifestes de la présence d'un contact anormal le long de la surface de discordance mésozoïque) sont des éléments conduisant à envisager pour ces formations un comportement visco-plastique, pouvant être celui d'un corps de Bingham, ou plus complexe tenant compte du frottement. Dans le cas d'un glissement sur une couche visco-plastique (fig. 12) de type Bingham à seuil de cisaillement $C_{p}$ formant un dièdre dont une face est horizontale, l'autre faisant un angle $\theta$ avec I'horizon, où '" est la longueur de la partie chevauchante non horizontale, $L$ la longueur totale du chevauchement, les calculs [24] montrent que:

$\frac{\ell}{L}=\frac{C_{p}}{\gamma h \sin \theta}$

Appliqués à ce modèle (bloc épais sur couche mince) afin de déterminer la longueur $\mathrm{L}$, pour $t=8 \mathrm{~km}, \theta=12^{\circ}$ et différentes valeurs de la cohésion $C_{p}$ comprises entre 2 et 50 bars, ils donnent les résultats du tableau 2.

L'absence de formation à comportement particulier nous amène à considérer cet ensemble comme une superposition d'un petit nombre de blocs beaucoup moins déformables que le niveau triasique dont les surfaces de contact sont des surfaces antérieures de stratification. Cinq surfaces majeures de glissement ont ainsi été mises en évidence dans cet ensemble (fig. 12), le long desquelles, sous l'hypothèse d'une cohésion structurale $C_{a}=30$ bars sur chacun de ces plans, on obtient, comme longueur de surfaces de décollement, les valeurs notées $x$ dans le tableau 3 .

Pour le niveau triasique, en prenant $C_{p}=15$ bars on obtient (tableau 2) une longueur L égale à $75 \mathrm{~km}$, cette valeur correspond à la distance de Gavarnie aux Sierras Marginales où Séguret [25], à l'aide d'autres considérations, situe l'amortissement frontal de la N.P. de Gavarnie.

\section{Conclusions}

Les mécanismes de déformation des terrains de la nappe de Gavarnie dépendent amplement des surfaces structurales antérieures, notamment de leur fréquence. IIs sont de type glissement structural continu pour le Paléozoïquie et de type glissement structural continudiscontinu pour le Crétacé, la liaison entre ces deux unités présentant une forte discontinuité.

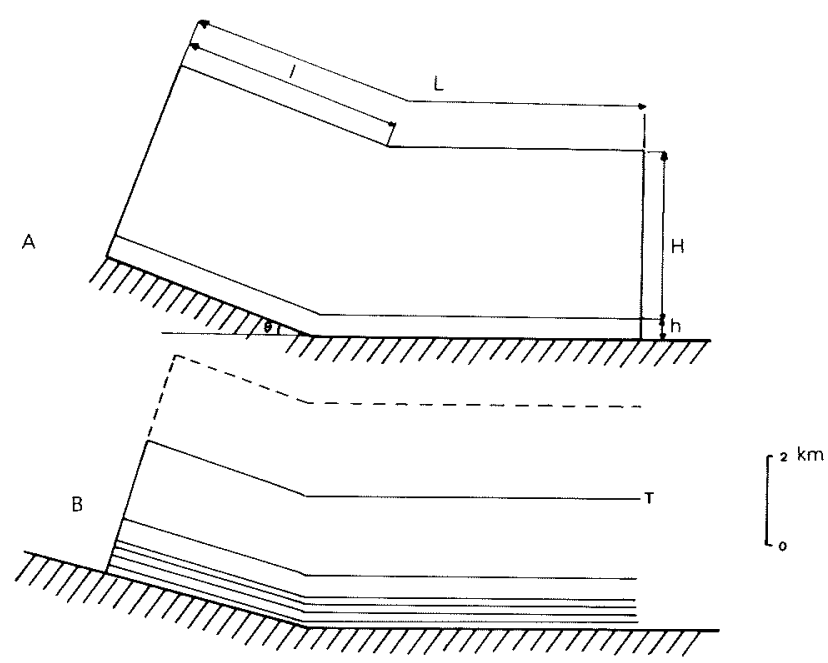

Fig. 12 Mise en place de la nappe Crétacé-Eocène

A Schéma géométrique. Déplacement d'ensemble sur le substratum par l'intermédiaire d'une couche visco-plastique (dont l'épaisseur $H$ est exagérée)

$B$ Surfaces de déplacement discontinu à l'intérieur du bloc précédent

$T$ : surface topographique actuelle ; en pointillé : partie érodée

Tableau I/ Longueur de la partie horizontale pour différentes valeurs de la cohésion

\begin{tabular}{|c|c|}
\hline $\begin{array}{c}\mathrm{C}_{\mathrm{p}} \\
\text { (bars) }\end{array}$ & $\begin{array}{c}\mathrm{L} \\
(\mathrm{km})\end{array}$ \\
\hline 50 & 22 \\
20 & 57 \\
10 & 114 \\
2 & 800 \\
\hline
\end{tabular}

Tableau /I/ Déplacements relatifs sur les surfaces de glissement (calcul)

\begin{tabular}{|c|c|}
\hline $\begin{array}{c}\text { Surface } \\
\text { de } \\
\text { glissement }\end{array}$ & $\begin{array}{c}x \\
(\mathrm{~km})\end{array}$ \\
\hline A & 12,1 \\
B & 11,8 \\
C & 11,5 \\
D & 11,4 \\
E & 10,7 \\
\hline
\end{tabular}

Le modèle obtenu à la suite de la détermination des déformations, résultant des mesures sur les structures induites observées, a permis d'estimer les déplacements relatifs de ces ensembles sous l'hypothèse de valeurs numériques raisonnables des constantes rhéologiques de la roche en mouvement.

Finalement, la tectogénèse de la nappe de Gavarnie se résume en une contraction régionale NS au cours de l'orogénèse alpine provoquant au $\mathrm{N}$, dans la zone de racine, un écoulement plastique ascendant des terrains paléozoïques situés entre deux ensembles rigides. La présence d'une couverture Crétacée dans la partie supérieure induit un écoulement vers le Sud. La 


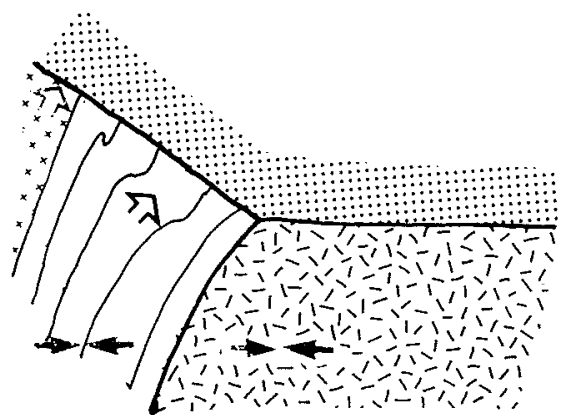

A

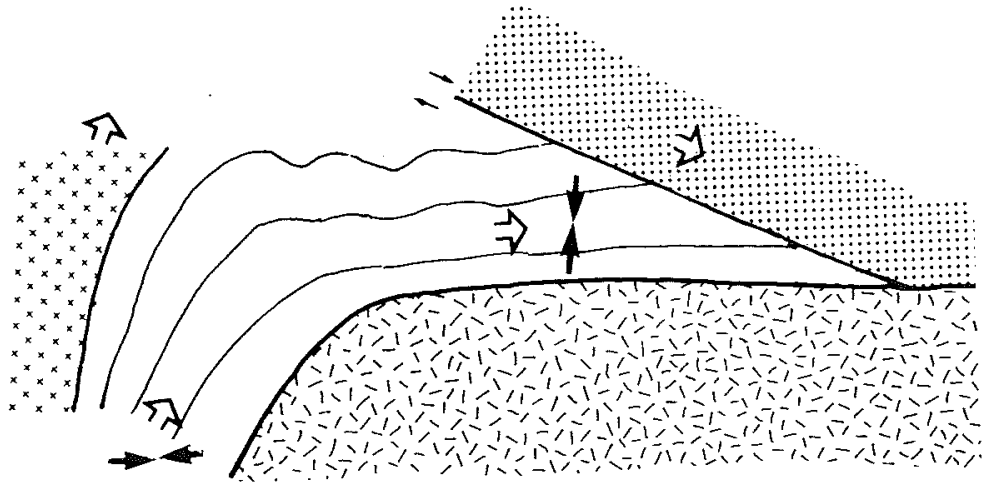

B

Fig. 13 Schéma de mise en place de la nappe paléozö̈que et de la nappe Crétacé-Eocène

A Stade initial

$B$ Stade final

(les flèches blanches indiquent les directions de déplacement, les flèches noires les directions de raccourcissement maximall)

mise en place de l'ensemble Paléozoïque-Crétacé s'effectue, pour le Crétacé gravitairement sur un dièdre à pentes de l'ordre de $12^{\circ}$ et horizontal, et pour le Paléozoïque sous l'effet de surcharges et d'entraînement tangentiel supérieur (fig. 13).

Ce schéma est applicable à d'autres structures chevauchantes célèbres telles que celles que l'on peut observer dans les montagnes Rocheuses, les Appalaches et les Alpes. II met en évidence les mécanismes de glissements structuraux sur des surfaces antérieures d'anisotropie (éventuellement courbes comme dans le cas du vaiont) fréquemment observés dans les glissements de terrain.

\section{Références bibliographiques}

[1] Bresson (A.). «Études sur les formations anciennes des Hautes et des Basses-Pyrénées (haute chaîne). " Bull. Carte Géol. Fr., XIV, 93, p. 45-322, 1903.

[2] Déramond (J.). «Déformation et déplacement des nappes : exemple de la nappe de Gavarnie (Pyrénées centrales). "Thèse Sciences, Toulouse, 409 p., 1979.

[3] Lamouroux (C.). «Les mylonites dans le massif de Néouvielle» (textures, déformations intracristallines). Thèse $3^{e}$ cycle, Toulouse, 148 p., 1976.

[4] Lamouroux (C.), Debat (P.), Déramond (J.), MajestéMenjoulas (C.). "Influence de massifs plutoniques hercyniens dans l'évolution des structures pyrénéennes: exemple du massif de Néouvielle. "Bull. Soc. Géol. Fr., (7) t. XXI, n² 2, p. 213-220, 1979.

[5] Majesté-Menjoulas (C.). «Évolution d'un segment de chaîne varisque : le Paléozoïque de la nappe de Gavarnie et le chevauchement de Cinq MontsGentiane. "Thèse Sciences, Toulouse, 331 p., 1979.

[6] Debat (P.). "Tectonique Tertiaire dans les formations métamorphiques des vallées de Gavarnie et Héas (Hautes-Pyrénées). " C.R. Somm. Soc. Géol. Fr., 2, p. 31-32, 1969.

[7] Déramond (J.), Rambach (J.M.). «Mesure de la déformation dans la nappe de Gavarnie : interprétation cinématique." Bull. Soc. Géol. Fr., (7), t. XXI, n ${ }^{\circ}$, p. 201-212, 1979.
[8] Déramond (J.), Litaize (D.). «Mesure informatique pour la détermination du taux de déformation." Applications. Bull. Soc. Géol. Fr., XVIII, 6, p. 1423-1434, 1976.

[9] Durney (D. W.). «Deformation history of the Western Helvetic Nappes, Valais; Switzerland. "Ph. D. Thesis, London University, 372 p., 1972.

[10] Durney (D.W.), Ramsay (J.G.). «Incremental strains mesured by syntectonic crystal growths. In Gravity and Tectonic " (de Jong, Scholten, ed.). Wiley et Sons, New-York, p. 67-95.

[11] Ramsay (J. G.). «The Measurement of strain and displacement in orogenic belts. "Geol. Soc. London, Sp. public. $n^{\circ} 3$, p. 43-79, 1969.

[12] Ramsay (J.G.), Graham (R.). "Strain variation in shear belts." Can. Journ. Earth. Sc., 7, p. 786-813, 1969.

[13] Inglès (J.), Déramond (J.). " Effet de frettage dans des séries rythmiques sédimentaires. "C.R. Acad. Sc., Paris, série D, 287, p. 1107-1110, 1978.

[14] Bucher (W.H.). «Role of gravity in orogenesis. " Bull. Soc. Geol. Am., 67, p. 1295-1318, 1956.

[15] Price (R.). "Large scale gravitational flow of supra-crustal rocks, Southern Canadian Rockies. In Gravity and Tectonics "(de Jong, Scholten, ed.); John Wiley et Sons, New-York, p. 491-502, 1973.

[16] Elliott (D.). "The motion of thrust sheets. " Journ. Geop. Res., 81, p. 949-963, 1976.

[17] Smoluchowski (M.S.). «Some remarks on the mechanics of over thrust. in Mechanics of thrust faults and decollement" (Barry Voight, ed.); Dowden, Hutchinson et Ross inc.; Stcondsburg. Pennsylvania, 1976.

[18] Hubbert (M.K.), Rubey (W.W.). "Role of fluid pressure in mechanics of overthrust faulting. "Bull. Geol. Soc. Am., 70, p. 115-166, 1959.

[19] Hsü (K.J.). "Role of cohesive strength in the mechanics of overthrust faulting and of lands liding. " Geol. Soc. Am. Bull., 80, p. 927-952, 1969.

[20] Nye (J.F.). «The mechanics of glacier flow.» Journ. Glac., 2, p. 82-91, p. 103-107, p. 339-341, 1952.

[21] Nye (J.F.). "The motion of ice sheets and glaciers. " Journ. Glac., 3, p. 493-507, 1959. 
[22] Nadai (A.). "Theory of flow and fracture of solids. » $2^{\mathrm{e}}$ éd., Mac Graw-Hill Book Co, Inc., New-York, London, 572 p., 1950.

[23] Kanizay (S. T.). «Mohr theory of stress and Prandlt compressed cell in relation to vertical tectonics. "U.S. Geol. Survey, Prof. Paper, 414 B, 1962.
[24] Rambach (J.M.), Déramond (J.). "Constant thickness overthrust on variable slope support." Tectonophysics, Letter Section, 60, T7-T16, 1980.

[25] Séguret (M.). "Étude tectonique des nappes et séries décollées de la partie centrale du versant $S$ des Pyrénées (caractères synsédimentaires, rôle de la compression et de la gravité)." Thèse Sciences, Montpellier, Ed. Ustela, 162 p., 1972. 